\title{
Posteriore EKG-Ableitungen bei Infarktverdacht
}

Ein Teil der akuten Myokardinfarkte entgeht dem herkömmlichen 12-KanalEKG. Um die elektrokardiografische Ausbeute zu optimieren, empfehlen sich zusätzlich EKG-Ableitungen.

- Um diese Forderung zu unterstreichen, wurde bei 53 Patienten, bei denen eine höhergradige Stenosierung im Bereich des Ramus circumflexus mittels Ballons dilatiert wurde, während der Koronarintervention ein 15-KanalEKG abgeleitet. Zusätzlich zu den üblichen EKG-Ableitungen wurden dabei drei weitere posteriore Ableitungen (V7-V9) dokumentiert. Dabei zeigte sich, dass Myokardischämien im Versorgungsgebiet des Ramus circumflexus nur über diese posterioren Ableitungen erfasst werden konnten.

\section{Kommentar}

Typische Vorderwand- oder Hinterwandinfarkte werden im Allgemeinen mittels 12-Kanal-EKG zuverlässig erfasst. Doch bei einem Drittel der Infarktpatienten wird der Infarkt nicht erkannt, wenn nicht zusätzliche EKG-Ableitungen erfolgen.
Dazu gehört der inferior-basale Hinterwandinfarkt, der durch einen Verschluss des Ramus circumflexus der linken Kranzarterie verursacht wird. Solche Infarkte können nur durch zusätzliche posteriore Ableitungen, nämlich von $V_{7}-V_{9}$, erkannt werden. Gleiches gilt auch für rechtsventrikuläre Infarkte, die nur erkannt werden, wenn zusätzlich rechtspräkordiale Ableitungen durchgeführt werden.

Fazit: Besteht bei einem Patienten dringender Infarktverdacht, und zeigt das 12-Kanal-EKG keine entsprechenden Veränderungen, so sollten immer zusätzliche posteriore und rechtskardiale Ableitungen durchgeführt werden.

P. StiefelhageN =
- R. A. Aqel et al.

Usefulness of three posterior chest leads for the detection of posterior wall acute myocardial infarction. Am. J. Cardiol. 103 (2009) 159-1642009) 1112-1116

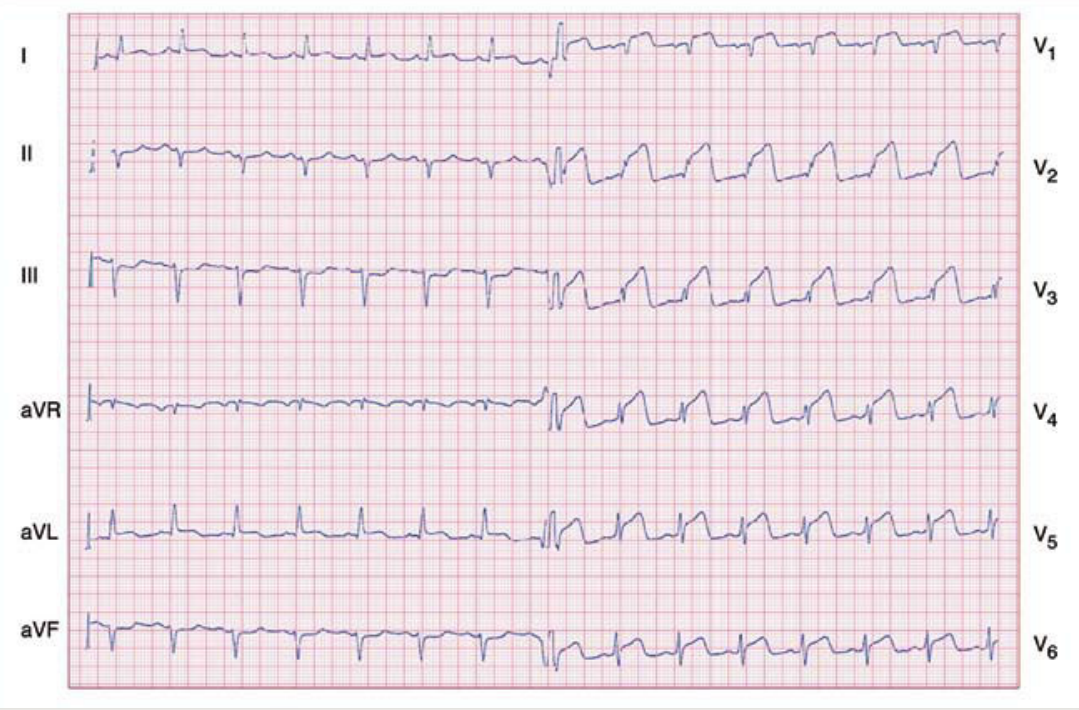

Bei den herkömmlichen EKG-Ableitungen wird ein Dritttel der Infarkte nicht erkannt. 